679

\section{CEREBROSPINAL FLUID OLIGOCLONAL BANDS AND IGG IN CHILDREN}

J. Paprocka1 ${ }^{1}$, E. Jamroz ${ }^{1}$, K. Szczechowska², J. Pytel ${ }^{2}$

${ }^{1}$ Child Neurology Department, ${ }^{2}$ Student's Scientific Society, Child Neurology Department, Silesian Medical University, Katowice, Poland

Introduction: Cerebrospinal fluid examination may be an essential aid in the diagnostic process of many neurological diseases in children as well as in adult patients. The detection of oligoclonal bands and the assessment of $\lg \mathrm{G}$ index contribute to information about the intrathecal production of oligoclonal lgG that may possibly occur in many neurological diseases. This includes mainly demyelinating and inflammatory diseases, but elevated IgG index and/or positive oligoclonal bands can be detected also in other conditions, such as neoplastic disorders or neurometabolic diseases/ progressive encephalopaties (PE). Intrathecal immunoglobulin synthesis can be measured either quantitatively, using the lgG index, or land qualitatively by oligoclonal bands detection.

Material and methods: Cerebrospinal fluid and serum samples from 287 patients hospitalized in Child Neurology Department Medical University of Silesia in Katowice in the years 2000-2009 were analyzed. The established diagnoses in examined group of patients included: demyelinating diseases (including MS, ADEM), neuroinfections (also neuroboreliosis), Guilliain -Barre syndrome (GBS), progressive encephalopathies, epilepsy, tumours of the central nervous system. Agarose gel electrophoresis was used for detection of CSF oligoclonal bands.

Results: Oligoclonal bands were detected in 108. In 50 patients with $\mathrm{PE}$, in 39 children with neuroinfections, Guilliain-Barre syndrome -13, tumours-2 patients.

Conclusions: Oligoclonal bands are important additional marker in different neurological disorders and can be used to confirm primary diagnosis.
680

PROGRESSIVE ENCEPHALOPATHIES IN CHILDREN

E. Jamroz ${ }^{1}$, J. Paprocka1 ${ }^{1}$ J. Pytel ${ }^{2}$, K. Szczechowska ${ }^{2}$

${ }^{1}$ Child Neurology Department, ${ }^{2}$ Student's Scientific Society, Child Neurology Department, Silesian Medical University, Katowice, Poland

Background: Progressive encephalopathies (PE) create a diverse group of neurological diseases, that may occur in children as well as in adults. Over the last twenty-five years the field PE has envolved from a limited group of rare, untreatable disorders to an important cause of acutely life-threating, but, for some number, treatable diseases. That is why the early establishment of the diagnosis is an essential issue also for genetic counselling.

Material and methods: Authors present a group of 256 children diagnosed with PE, hospitalized over years 1999 - 2009 in Child Neurology Department, Medical University of Silesia, Katowice, Poland.

Results: Based on performed metabolic and genetic work-up (metabolic screening, neuropathological examinations, neuroimaging studies, molecular tests) in 127 patients leukodystrophies of known origin were diagnosed. In 37 cases lysosomal storage diseases were recognized, 24 children suffered from mitochondrial diseases, peroxisomal diseases occurred in 9 patients. Disorders of intermediatory metabolism (aminoacidopathies, organic acidurias, disorders of ammonia detoxification, disorders of fatty acid oxidation, of carbohydrate metabolism) were found in 50 children. Remaining 7 patients were diagnosed with other encephalopathies (f.e. Pelizaeus-Merzbacher disease, MLC, GLUT-1)

Conclusions: Due to the wide range of biochemical, histological and molecular study provided and symptoms-based approach in 127 children with $\mathrm{PE}$ it was possible to establish the appropriate diagnoses. 